\title{
Jean Sgard, Crébillon fils, le libertin moraliste
}

\section{Aurelio Principato}

\section{(2) OpenEdition}

\section{Journals}

\section{Edizione digitale}

URL: http://journals.openedition.org/studifrancesi/35612

DOI: 10.4000/studifrancesi.35612

ISSN: 2427-5856

\section{Editore}

Rosenberg \& Sellier

\section{Edizione cartacea}

Data di pubblicazione: 1 juillet 2005

Paginazione: 118-121

ISSN: 0039-2944

\section{Notizia bibliografica digitale}

Aurelio Principato, «Jean Sgard, Crébillon fils, le libertin moraliste», Studi Francesi [Online], 145 (XLIX | I) 2005, online dal 01 novembre 2015, consultato il 19 avril 2021. URL: http://journals.openedition.org/ studifrancesi/35612 ; DOI: https://doi.org/10.4000/studifrancesi.35612

\section{Questo documento è stato generato automaticamente il 19 avril 2021.}

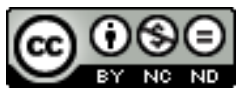

Studi Francesi è distribuita con Licenza Creative Commons Attribuzione - Non commerciale - Non opere derivate 4.0 Internazionale. 


\title{
Jean Sgard, Crébillon fils, le libertin moraliste
}

\author{
Aurelio Principato
}

\section{NOTIZIA}

JEAN SGARD, Crébillon fils, le libertin moraliste, Paris, Desjonquères, 2002, pp. 315.

1 Nell'ambito del contributo ancor un più ampio da lui apportato, Jean Sgard acquisiva agli studi settecenteschi francesi con il volume Prévost romancier (1968) una massa di informazioni storico-letterarie che si erano poi riverberate nel grande sviluppo critico degli ultimi trent'anni. Mentre, nel caso di Prévost, la prima edizione moderna di un vasto continente narrativo aveva fatto seguito alla riscoperta, questa nuova biografia di Crébillon riassume e conclude la pubblicazione delle Oeuvres complètes, diretta sempre da Sgard per i Classiques Garnier. E, grazie al progresso delle conoscenze, si configura in modo del tutto diverso rispetto alla grande monografia citata, così come, per altri versi, supera la precedente ricerca specifica, cioè quella di H.-G. Funke (Crébillon fils als Moralist und Gesellschaftskritiker, Heidelberg, 1972). L'accumulo di documentazione non è più necessario, e d'altra parte non si conformerebbe alle caratteristiche del romanziere ora esplorato con lo stesso amore e con la stessa lucidità critica. Infatti, non solo le notizie che si hanno su Crébillon sono ancora più scarse di quanto non lo fossero per Prévost, ma la sua narrativa riveste quell'elegante leggerezza che già si opposero, nel secondo terzo del XVIII secolo, alla profondità passionale dell'autore Cleveland e di Manon Lescaut. E dell'eleganza di Claude Crébillon si riveste precisamente il libro di Sgard, con una agilità e un sapore che avevamo potuto già apprezzare in un successivo volume su Prévost (L'Abbé Prévost, labyrinthes de la mémoire, P.U.F., 1986) e in altri innumerevoli più brevi interventi.

2 Nel rendere conto di questo nuovo libro, cercheremo dunque di riferirci costantemente ai due dati essenziali sopra indicati dal primo, la scarsità di informazioni, discende la domanda preliminare che si pone l'autore sul senso della sua fatica: ricostruire la vita e 
la carriera di Crébillon equivale a interpretare costantemente, a spiegare il senso della sua ricerca stilistica e della ricerca morale connotata dal libertinage. Sgard ci riesce esplorando con straordinaria finezza anche i documenti notarili. Per primo il testamento, che ci fornisce i dati di base sui quali si orientano poi le pagine successive: il nome, il padre, gli amici, la funzione di censore raggiunta nella maturità. Il nome ci conduce infatti allo statuto sociale dello scrittore, alla sua appartenenza borghese e alle aspirazioni nobiliari, proprie del contesto storico in cui visse, ma anche al rapporto tormentato e contraddittorio con le società galanti in cui si trovò introdotto, senza condividerne mai sostanzialmente la mentalità. Da qui la lettura della sua vocazione di romanziere libertino, che lo ha consacrato come eponimo di tutto un filone letterario: secondo una linea già percorsa da Siemek (1981), Sgard ci propone il ritratto di un autentico moralista ma rapportandosi come è suo costume, prima ancora che all'opera narrativa, alle motivazioni intime che egli recupera attraverso il paradigma indiziario degli atteggiamenti esistenziali.

Quanto al rapporto con il padre Prosper, reso emblematico dall'appellativo «fils» che ha tradizionalmente accompagnato il nome di Crébillon, Sgard nota preliminarmente come nelle succinte testimonianze biografiche e nelle caratterizzazioni più tarde si giochi su di esso buona parte di ciò che egli felicemente chiama «rhétorique du contraste». Opporre il padre al figlio, significa infatti accentuarne la differente indole, la natura antitetica del rispettivo successo, per di più ottenuto in due diversi ambiti letterari. La gloria del drammaturgo Prosper presso i contemporanei, che fece tanta ombra a Voltaire, servì a bollare la licenziosità del romanziere Claude, così come oggi, rovesciando la simmetria, interessarsi all'appeal erotico della sua narrativa serve ad esaltare l'attualità di un genere emergente che prende il sopravvento sulle fosche tinte tragedia classica.

4 Eppure, la vocazione teatrale fu tutt'altro che assente nel figlio (cap. III, «La folie du théâtre»). Abbiamo la certezza della sua frequentazione degli ambienti della scena, in particolare della compagnia degli «italiens», e possiamo intravedere la sua collaborazione alle parodie di Giovanni Antonio Romagnesi, delle quali Sgard ci offre un gustoso campionario. Tali debutti si legano a una particolare atmosfera di appuntamenti di taverna dove si improvvisavano strofette cantate su arie note (i «ponts-neufs»), protagonisti gli amici che si riunivano dal 1729 al «Caveau» (cap. IV: «L'envol»), e che comprendeva oltre a Piron, Duclos, Moncrif e altri, i due Crébillon, così accomunati dal gusto della gioiosa convivialità, e Collé, che rimarrà legato per tutta la vita al nostro autore. In siffatto ambiente si sarebbe determinata la formazione stilistica di Claude, in chiave di contrapposizione ai generi elevati.

5 È stato possibile ricostruire il comporsi e ricomporsi di queste «sociétés badines», quali fiorirono lungo tutto l'arco del secolo, venendo a essere confuse troppo facilmente in un'unica tipologia nel secolo seguente e, per altri versi, irrigidite in una classificazione quasi istituzionale (si veda per questo la nostra introduzione a due «dialogues des morts» di Crébillon, nel secondo volume delle CEuvres complètes sopra citate). I contorni di esse sono molto più sfumati e mutevoli, anche se la naturale parodia delle Accademie serie può aver facilitato tale schematismo. Di fatto sappiamo che l'intrufolarsi nel Caveau di un uomo politicamente rilevante, per quanto gaudente, come Maurepas tolse presto spontaneità alle riunioni, determinandone la fine, e possiamo domandarci se situare in questo momento anche lo scontro tra padre e figlio (ma Sgard preferisce differire la crisi dei loro rapporti a dopo il 1750). È opportuno invece rilevare come 
alcuni dei giovani animatori dei Caveau si ritrovino più o meno regolarmente a pranzo, a qualche anno dopo, presso l'ex-attrice Mlle Quinault, a spese del conte di Caylus. Questi ultimi due, con lo stesso Maurepas, avevano dato vita diverso tempo addietro a una società di «teatromani», come hanno dimostrato gli studi recenti di Judith Curtis e D. Trott ${ }^{1}$. Tuttavia, assieme alla continuità posta sotto il segno dei «lazzi» e della Comédie italienne, va rilevata la funzione sociale molto diversa di tali divertimenti, in cui la partecipazione diretta o indiretta dei potenti poteva verosimilmente assumere una funzione di controllo politico.

Nell'attesa di brillare per alcuni mesi nel salotto di Mlle Quinault, Crébillon ha tuttavia iniziato nel modo più felice la sua carriera di rornanziere. La sua prima opera pubblicata, Le Sylphe (1729 o 1730) annuncia quella propensione per l'incantesimo erotico che si svilupperà con modalità diverse in Tanzaï et Néadarné (1734) e nel Sopha. Per il primo romanzo (che ci si abituò presto, ma arbitrariamente, a chiamare L'Écumoire), sulla leggerezza fiabesca prevale infatti il «peso» dell'allegoria politica, che causò la prigione allo scrittore. E per il secondo, la cui pubblicazione apportò a Crébillon danni non meno seri (l'esilio da Parigi), si mette in gioco in modo altrettanto chiaro il rapporto complesso che lega il racconto libertino alla critica sociale se non alla satira del potere. Sgard, che intanto non ha tralasciato, nel racconto della vita di Crébillon, le tracce della sua frequentazione di Voltaire, relativamente a Le Temple du goût (situazione non estranea, neanche in questo caso, al ruolo del padre Prosper, che svolgeva parimenti le funzioni di censore), mostra in modo pienamente convincente come non si giustifichi una lettura di Tanzaï in chiave giansenista.

7 Su un altro versante, in questo primo decennio di carriera, abbiamo les Lettres de la Marquise e Les Égarements du coeur et de l'esprit, romanzi privi di trasposizione magico orientale, e connessi invece a una tradizione di carattere drammatico passionale filtrata, nel secolo classico, dalle Lettres Portugaises e da Racine. Di ciò si ritrovano gli echi visibili nel primo romanzo, ma trascritti in uno stile personale del quale Sgard, lungo tutto il libro, rileva le potenzialità musicali, e, nel caso specifico, «ce déroulement fluide de la phrase, soutenu par de discrètes scansions», analogo a quello che realizzava Couperin sul clavicembalo negli stessi anni. Si intravede anche la possibile influenza della recitazione di Jeanne Gaussin, l'attrice raciniana alla quale Crébillon è contemporaneamente legato.

Gli Égarements segnano il culmine della produzione degli anni '30. Ma esistono già versioni primitive di opere che appariranno molti anni dopo, o che segneranno il suo destino, come il Sopha. Le prime tracce della circolazione clandestina di questo romanzo sono infatti ben anteriori al gennaio 1742, data della pubblicazione, e Fougeret de Monbron aveva avuto il tempo di plagiarlo nel Canapé couleur de feu. Lo scandalo che esso provoca attira i fulmini del cancelliere d'Aguesseau, che, come sappiamo, aveva proscritto romanzi e periodici nel 1737. Per Crébillon fu l'esilio a trenta leghe da Parigi, e se questo durerà solo tre mesi, gli occorreranno tre anni almeno per risalire la china dei rapporti sociali, ma molti di più per persuaderlo a interrompere il suo silenzio di scrittore. L'episodio è cruciale anche perché mette in gioco il rapporto tra letteratura $\mathrm{e}$ potere politico, e con esso il problema del libertinage. Sgard scioglie in modo definitivo la confusione dei due piani, sottolineando come condannata non fosse tanto l'immoralità quanto la potenziale identificazione dei personaggi e delle storie con figure e situazioni reali, e come il divario fra il libertinaggio effettivo e l'elegante analisi sociale e psicologica di Crébillon sia tale da meritare di sottrargli la sua cattiva 
reputazione. E ciò si traduce ovviamente in una nuova e diversa presenza del romanziere nell'attenzione critica di oggi. Ma, all'epoca, la riunione dei due termini del sottotitolo (conte moral) sovvertiva le attese, e Crébillon ne fu del tutto cosciente.

Fino alla pubblicazione (1754) di Ah, quel conte!, rimasto lungamente nel cassetto, il silenzio di Crébillon è rotto soltanto da due dialoghi dei morti inseriti nel collettivo Recueil de ces Messieurs, e forse, nello stesso anno 1745, da una trasparentissima satira politica, Les Amours de Zéokinizul [=Louis XV] roi des Kofirans [=François]. In mancanza di prove contrarie Sgard ha inserito questo testo Delle CEuvres complètes, ritenendo che si possa mantenerne l'attribuzione, anche se ciò presuppone un'audacia oltremodo recidiva nello scrittore. Ma bastava forse a garantirlo la protezione di Maurepas conosciuto ai tempi del Caveau. A Maurepas era del resto strettamente legato il conte di Caylus, nel cui ambiente si muove Crébillon, partecipando ai «dîners» di Mlle Quinault, e, appunto, al Recueil de ces Messieurs che ne costituisce il frutto letterario.

Entra qui in campo (cap. VII e VIII) la testimonianza di Mme de Graffigny, la cui corrispondenza, recentemente pubblicata da un'équipe di ricercatori canadesi presso la Voltaire Foundation, ha fatto subire un balzo capitale alla nostra conoscenza della vita letteraria parigina di quel decennio. Françoise de Graffigny, che pubblica la sua prima novella nello stesso Recueil, ci dà indizi su altre operette mai pubblicate (un terzo dialogo dei morti, diversi aneddoti galanti, una «Chanson des pendus» contro Cahusac, una Marianne courtisane che è una palese parodia di Marivaux). La scrittrice ci dà soprattutto la chiave per decifrare il «malaise» dello scrittore, che si dibatte tra ambizioni frustrate, difficoltà economiche, attrattiva su di lui esercitata dai salotti altolocati, rapporto contrastato con il caratteriale Caylus, il quale lo vezzeggia ma è molto diverso per estrazione sociale e contemporaneo dilettarsi plebeo. Finalmente, dopo aver abitato con Mme de Saint-Maur, flirtato con la baronessa di Preysing, frequentato privatamente Mme de Graffigny, Crébillon sposa nientemeno una donna d'alto lignaggio, Marie-Henriette de Stafford, che conosce nel 1744 negli ambienti stuartisti. Sembra la fine dei suoi affanni, ma non è cosi: lo scrittore non raggiungerà la tranquillità economica, né il desiderato posto di rango in società. Tutta la vicenda, ricostruita da Sgard a stretto contatto con Philip Stewart, costituisce una delle maggiori acquisizioni inerenti alla biografia di Crébillon. Il matrimonio è celebrato quasi di nascosto nel 1748 (nel frattempo era nato anche un figlio morto quasi subito), e dopo qualche anno svaniscono le speranze di una ricca eredità. Da qui le peregrinazioni della coppia in diverse dimore, in preda ad esigenze di sostentamento, e in particolare a Sens, cioè fuori Parigi. Sul piano esistenziale, si tratta di una apparente conversione alla vita familiare alla quale i contemporanei stentarono a prestar fede.

11 In un appartamento del castello di Saint-Germain, dove la coppia si trasferisce nel 1752, Crébillon riprende la sua attività di scrittore, pubblicando Ah, quel conte! e i «contes dialogués», scrivendo Les Heureux Orphelins, collaborando al periodico «La Bigarrure». La moglie muore, egli è costretto a vendere la sua biblioteca (ciò che ci permette di possederne l'inventario, e le interessanti indicazioni che ne conseguono). Ma non cessa la sua ricerca sullo stile, come si evince dalle opere della maturità, che risentono del successo di Richardson. Si rinnovano le frequentazioni della «bonne compagnie» parigina e delle sue costellazioni variamente denominate (secondo Caveau, «Société Dominicale»...), dove il potere economico dei «financiers» ha un peso sempre maggiore. Nel 1760 è nominato censore, come lo è stato suo padre, che muore due anni dopo. Sgard coglie l'occasione per illustrare il valore di un tale ruolo all'epoca, orientandoci 
sul modo in cui Crébillon lo esercitò. In particolre egli dà avviso favorevole al Barbier de Séville di Beaumarchais (1775), e prende partito per la magistratura contro la riforma di Maupeou.

12 La carriera dello scrittore si chiude con le Lettres de la Duchesse e con le Lettres athéniennes, che recuperano e accentuano il registro tragico-passionale, già presente, come abbiamo visto, nei primi romanzi e tuttavia misconosciuto, in quanto, anche all'estero, di Crébillon si era ormai consolidata la fama di scrittore francese libertino per eccellenza. In virtù della stessa nomea, passarono sotto il suo nome numerosi testi licenziosi pur lontanissimi dalla sua ispirazione.

L'ultimo capitolo della biografia è dedicato agli ultimi giorni di Claude Crébillon e al bilancio che si può trarre della sua esistenza a partire dal semplice inventario dei suoi beni, indizi che Sgard torna ad incrociare magistralmente con tutti quelli che gli provengono dalla sua vita e dalle sue opere. Ci resta, con l'impronta del grande scrittore, l'impressione acuta delle difficoltà quotidiane che la vita settecentesca riservava a tale professione. Anche per questi versi, il libro di Sgard è esemplare.

\section{NOTE}

1. Introduction à l'Histoire et Recueil des Lazzis (SVEC 338, 1996). 